\title{
Detailed photometric analysis of young star groups in the galaxy NGC 300*
}

\author{
M. J. Rodríguez ${ }^{1}$, G. Baume ${ }^{1,2}$, and C. Feinstein ${ }^{1,2}$ \\ ${ }^{1}$ Instituto de Astrofísica de La Plata (CONICET-UNLP), Paseo del bosque S/N, La Plata (B1900FWA), Argentina \\ e-mail: jimenaro@fcaglp.unlp.edu.ar \\ 2 Facultad de Ciencias Astronómicas y Geofísicas, Universidad Nacional de La Plata, Paseo del bosque S/N, La Plata (B1900FWA), \\ Argentina
}

Received 2 December 2015 / Accepted 10 August 2016

\begin{abstract}
Aims. The purpose of this work is to understand the global characteristics of the stellar populations in NGC 300. In particular, we focused our attention on searching young star groups and study their hierarchical organization. The proximity and orientation of this Sculptor Group galaxy make it an ideal candidate for this study.

Methods. The research was conducted using archival point spread function (PSF) fitting photometry measured from images in multiple bands obtained with the Advanced Camera for Surveys of the Hubble Space Telescope (ACS/HST). Using the path linkage criterion (PLC), we cataloged young star groups and analyzed them from the observation of individual stars in the galaxy NGC 300 . We also built stellar density maps from the bluest stars and applied the SExtractor code to identify overdensities. This method provided an additional tool for the detection of young stellar structures. By plotting isocontours over the density maps and comparing the two methods, we could infer and delineate the hierarchical structure of the blue population in the galaxy. For each region of a detected young star group, we estimated the size and derived the radial surface density profiles for stellar populations of different color (blue and red). A statistical decontamination of field stars was performed for each region. In this way it was possible to build the colormagnitude diagrams (CMD) and compare them with theoretical evolutionary models. We also constrained the present-day mass function (PDMF) per group by estimating a value for its slope.

Results. The blue population distribution in NGC 300 clearly follows the spiral arms of the galaxy, showing a hierarchical behavior in which the larger and loosely distributed structures split into more compact and denser ones over several density levels. We created a catalog of 1147 young star groups in six fields of the galaxy NGC 300, in which we present their fundamental characteristics. The mean and the mode radius values obtained from the size distribution are both $25 \mathrm{pc}$, in agreement with the value for the Local Group and nearby galaxies. Additionally, we found an average PDMF slope that is compatible with the Salpeter value.
\end{abstract}

Key words. stars: luminosity function, mass function - galaxies: individual: NGC 300 - galaxies: star clusters: general galaxies: star formation

\section{Introduction}

The distribution and properties of stellar clusters and starforming complexes provide valuable information that helps understanding the history of the star formation inside galaxies and the way this is related with the dynamic and chemical enrichment of each system. This reveals the importance of performing global studies and assembling catalogs of clusters over the nearest galaxies.

The galaxy NGC 300 is the brightest of five main spiral galaxies forming the Sculptor Group. It is a spiral galaxy Sc D (McConnachie 2012) and presents several regions of massive star formation. This galaxy also has an orientation that minimizes the absorption effects, and it is located close enough to identify stellar clusters and their individual members by using images from telescopes with excellent angular resolution. In particular, Pietrzyński et al. (2001) have provided a catalog with over one hundred OB associations in this galaxy using the path linkage criterion (PLC) and ground-based observations obtained with the ESO/MPI 2.2 m telescope.

* Full Table 2 is only available at the CDS via anonymous ftp to cdsarc.u-strasbg.fr (130.79.128.5) or via

http://cdsarc.u-strasbg.fr/viz-bin/qcat?]/A+A/594/A34
The aim of this work is to catalog and analyze the young star groups in NGC 300 using excellent quality data from the Hubble Space Telescope (HST). In particular, the Advanced Camera for Surveys (ACS) provides the quality we require, and some of the observed fields cover an important part of the galaxy NGC 300 (Bresolin et al. 2005). These data will allow us to obtain much more detailed information for the stellar groups than groundbased observations, such as the corresponding radial profiles and photometric diagrams. All these tools and their comparison with theoretical models will help us understand the properties of their individual populations.

The paper is organized as follows: in Sect. 2 we describe the observations and data sets. The reduction, astrometric corrections, and correlation procedure of the data are presented in Sect. 3. Section 4 discusses the method we used to search for and identify stellar groups. Section 5 presents our analysis of the detected associations. We discuss our results concerning the size, mass function, and hierarchical behavior in Sect. 6. Finally, in Sect. 7 we summarize our results.

\section{Data and observation}

The data used in this work, images, and photometric tables correspond to the "star files" from the ACS Nearby Galaxy Survey 


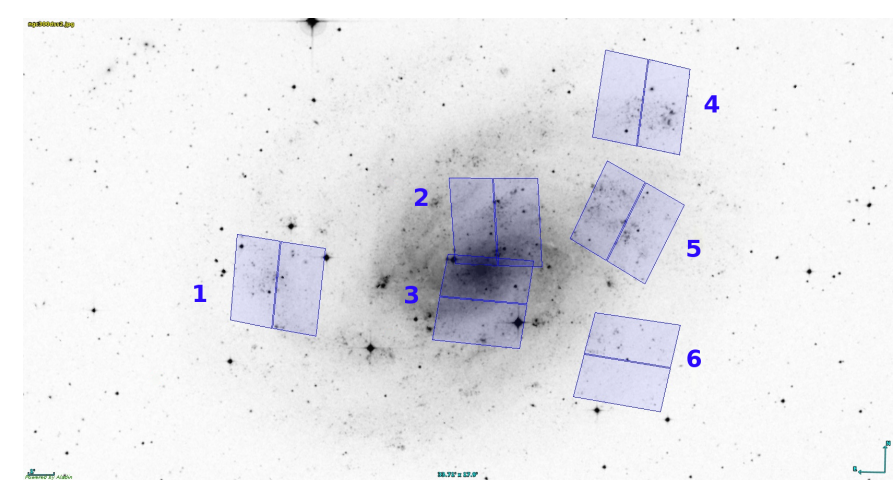

Fig. 1. Distribution of the different fields used in this work overlaid on a DSS image of NGC 300.

(ANGST). They were obtained from the database of the Space Telescope Science Institute (STScI) ${ }^{1}$. These files contain the photometry of all objects classified as stars with good signalto-noise values $(S / N>4)$ and data flag $<8$. The observations were carried out with the camera ACS/HST. They correspond to six fields in NGC 300 (see Fig. 1) and were obtained during HST Cycle 11 as part of program GO-9492 (PI: F. Bresolin). These observations employed the Wide Field Camera (WFC) of the ACS, obtaining exposures of $360 \mathrm{~s}$, three in the $F 435 \mathrm{~W}$ and $F 555 \mathrm{~W}$ bands and four exposures in the $F 814 \mathrm{~W}$ band, over each field. The WFC has a mosaic of two CCD detectors and a scale of $0.049^{\prime \prime} /$ pixel, the field of view covered is $3.3^{\prime} \times 3.3^{\prime}$.

At the considered distance of $1.93 \mathrm{Mpc}$ (Bresolin et al. 2005), the following relationship applies: $1^{\prime \prime}=20$ pix $\sim 9.4 \mathrm{pc}$.

\section{Photometry and astrometry}

\subsection{Photometry}

The binary fits tables of photometry as defined in Dalcanton et al. (2008) were obtained from the STScI data base. The high image resolution allows stellar point spread function (PSF) photometry in nearby galaxies. This enables studying their different stellar populations in great detail. The photometry was carried out using the package DOLPHOT adapted for the ACS camera (Dolphin 2000).

\subsection{Astrometry}

Using the $\mathrm{ALADIN}^{2}$ tool to compare the images of each observed NGC 300 field with the respective Dalcanton et al. (2008) catalog, a systematic difference (see Table 1) was found between the World Coordinate System (WCS) indicated in the image headers and those given by the catalog. Then, we compared the positions with the astrometric catalogs UCAC 4 and GSC 2.3 and verified that the WCS coordinates were correct. Next we accurately evaluated the difference between the two coordinate systems. To do this, we used the task xy2rd of the IRAF's ${ }^{3}$ stsdas package to obtain the WCS coordinates of some (see Table 1) bright and unsaturated stars in the $F 555 \mathrm{~W}$ band images of each field. We compared these values with the corresponding ones given by Dalcanton et al. (2008). These corrections were applied to all the objects in this catalog. Finally, we compared each

\footnotetext{
1 MAST: https://archive.stsci.edu/; https://hla.stsci. edu/

2 http://aladin.u-strasbg.fr/

3 IRAF is distributed by NOAO, which is operated by AURA under cooperative agreement with the NSF.
}

Table 1. Corrections applied to the coordinates given by Dalcanton et al. (2008).

\begin{tabular}{cccc}
\hline \hline Frame & $\Delta \alpha \cos (\delta)\left[{ }^{\prime \prime}\right]$ & $\Delta \delta\left[{ }^{\prime \prime}\right]$ & $\mathrm{N}$ \\
\hline 1 & $3.283 \pm 0.024$ & $-1.434 \pm 0.044$ & 5 \\
2 & $0.335 \pm 0.021$ & $-2.316 \pm 0.087$ & 3 \\
3 & $-1.247 \pm 0.032$ & $-0.462 \pm 0.050$ & 3 \\
4 & $0.519 \pm 0.027$ & $-2.541 \pm 0.078$ & 3 \\
5 & $-0.355 \pm 0.018$ & $0.886 \pm 0.007$ & 6 \\
6 & $-0.021 \pm 0.004$ & $0.029 \pm 0.009$ & 7 \\
\hline
\end{tabular}

Notes. In Col. 1 we indicate the frame ID, the corresponding corrections are given in Cols. 2 and 3. Column 4 lists the number of stars used in the coordinate alignment procedure.

resulting catalog with its corresponding image using ALADIN. The catalogs and images agreed well, although we note that one small relative distortion $\left(\sim 0.1^{\prime \prime}\right)$ still prevailed over some fields.

\subsection{Catalog correlation}

The catalogs given by Dalcanton et al. (2008) provide photometric information through three tables for each field, giving in each one the magnitudes of only two bands for each object. We therefore constructed a catalog with the three photometry bands for each field from them. This was performed with the STILTS 4 cross-correlation (with logical "OR") between tables with $F 555 W-F 435 W$ bands and those with $F 814 W-F 555 W$ bands.

Furthermore, fields 2 and 3 slightly overlap (see Fig. 1), therefore we took a final magnitude for the stars in the overlap region that results from the average between the stars in each field. Using STILTS, we then joined the information and list the two fields as one single field in the table.

\section{Searching for young stellar groups}

To evaluate the completeness of the data, we built the luminosity function for each table (see Fig. 3). The number of stars per bin begins to decrease below a magnitude $F 555 W=27$. Therefore we considered that the sample is complete for $F 555 W<26.5$.

For better identification throughout the text, we distinguish the following four groups of stars:

- the blue group: $F 435 W-F 555 W<0.25$ and $F 555 W-$ $F 814 W<0.25$;

- the blue bright group: $F 555 W<25, F 435 W-F 555 W<$ 0.25 and $F 555 W-F 814 W<0.25$;

- the red group: $F 435 W-F 555 W>0.6$ and $F 555 W-$ $F 814 W>0.6$;

- the red bright group: $F 555 W<25, F 435 W-F 555 W>0.6$ and $F 555 W-F 814 W>0.6$.

In Fig. 2 we show the CMDs of all the stars in the six fields, indicating the previous groups of stars and the location of particular isochrones for solar metallicity (Marigo et al. 2008; Girardi et al. 2010). For the blue bright sample at $F 555 W=25$, the oldest main-sequence turn-off corresponds to $235 \mathrm{Myr}$, and the lowest stellar mass value for a main-sequence star is $\sim 7.4 M_{\odot}$.

We used the selected blue bright group to detect young stellar structures, in particular OB associations and young stellar clusters. For this task, we applied different methods that we describe in Sects. 4.1 and 4.2

4 http://www.star.bris.ac.uk/ mbt/stilts/ 

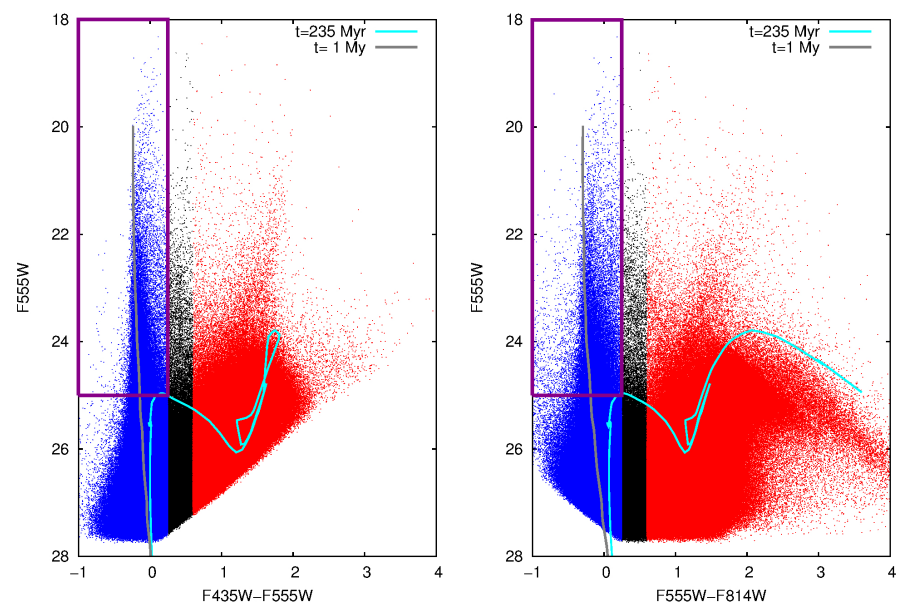

Fig. 2. Total CMDs of the six NGC 300 fields with indicative isochrones with solar metallicity (see Sect. 5.4) corresponding to 1 Myr (grey line) and $235 \mathrm{Myr}$ (turquoise line). Inside the rectangle we plot the stars belonging to the blue bright group; red and blue show the red and blue group.

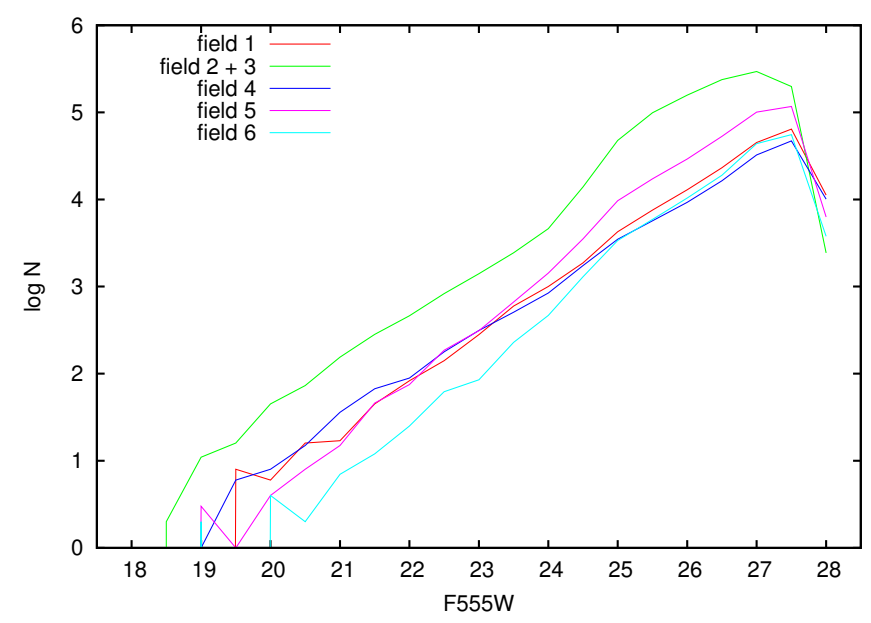

Fig. 3. Luminosity functions for the studied fields (fields 2 and 3 are reported as one single field).

\subsection{Stellar density maps}

With the aim of better understanding the distribution of different stellar populations in the galaxy and to have an additional tool to identify young star groups, we used the blue bright group and the red bright group to build spatial density stellar maps for the observed regions. In this process, we constructed for each field a two-dimensional histogram counting the numbers of stars in spatial bin sizes of 8.0 arcsec. Then we applied the drizzle method (Fruchter \& Hook 2002) considering a 2.0 arcsec step. The obtained density maps (six) were combined into one image with astrometrical calibration.

Then, we used the Source Extractor code (SExtractor) $^{5}$ on the obtained blue stellar density map to identify overdensities that could be identified as young stellar groups. To do this we adopted the default convolution mask of two pixels and assumed a size of four pixels for the background mesh. A larger mesh would imply that the background of the density stellar maps would be too smooth, and therefore some sources might be lost. A detection threshold of $1.5 \sigma$ above the local background was

\footnotetext{
5 http://sextractor. sourceforge.net/
}

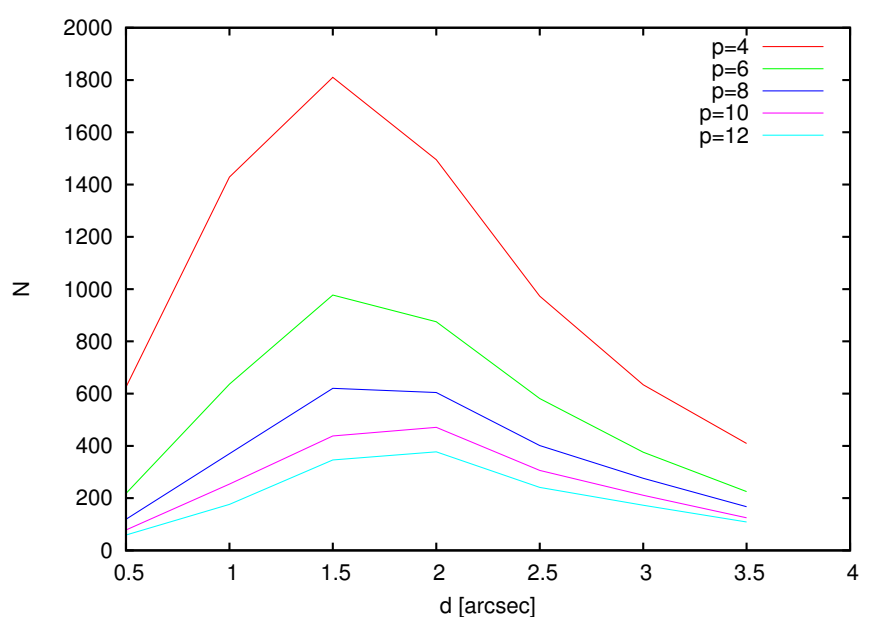

Fig. 4. Behavior of the number of groups detected using the PLC technique with respect to the parameter $d_{\mathrm{s}}$ for different values of the parameter $p$.

adopted, and objects with more than four pixels were considered as reliable overdensities. We assumed a value for the deblending contrast parameter $\delta_{\mathrm{c}}=0.001$ because this gives the best separation between sources.

The application of this method resulted in the detection of 289 candidates for young groups.

\subsection{Path linkage criterion}

We also employed the PLC technique (Battinelli 1991) to identify young star groups in NGC 300. This method consists of connecting blue stars that are less distant than a fixed parameter called $d_{\mathrm{s}}$. When it is possible to link more than $p$ stars, we have a candidate $\mathrm{OB}$ association or stellar clusters.

We applied this method to the selected blue bright group of stars on each covered field. Then, we had to choose adequate values for the parameters $p$ and $d_{\mathrm{s}}$. The lowest value of the number of stars that a group should have to be considered an association or stellar cluster $(p)$ is a parameter difficult to establish, since a low value results in many spurious detections, while with a high value the smallest groups might be lost. We studied the number of groups identified using PLC with respect to the parameter $d_{\mathrm{s}}$ for different values of $p$ (see Fig. 4). Finally, we considered that ten stars is a reasonable value for the parameter $p$ and adopted a value range between $0.5-2.5 \operatorname{arcsec}$ for $d_{\mathrm{s}}$. The advantage of starting the search with a small distance is to detect the smallest subgroups of each large association or stellar complex that otherwise would have been lost in larger structures. After detecting the smallest groups, the star members of these groups were eliminated from the sample and the PLC was run again, but increasing the value of $d_{\mathrm{s}}$. This procedure was repeated until $d_{\mathrm{s}}$ reached 2.5 arcsec. Using this method, we detected the individual groups but not the form in which they merge into larger structures. We discuss this point in Sect. 6.2.

Using the PLC technique, we detected 1147 young stellar groups in the six fields.

\subsection{Results of the search}

All the OB associations detected by Pietrzyński et al. (2001) and located in the six ACS fields were found with both techniques used in this work. The obtained detections with the different 


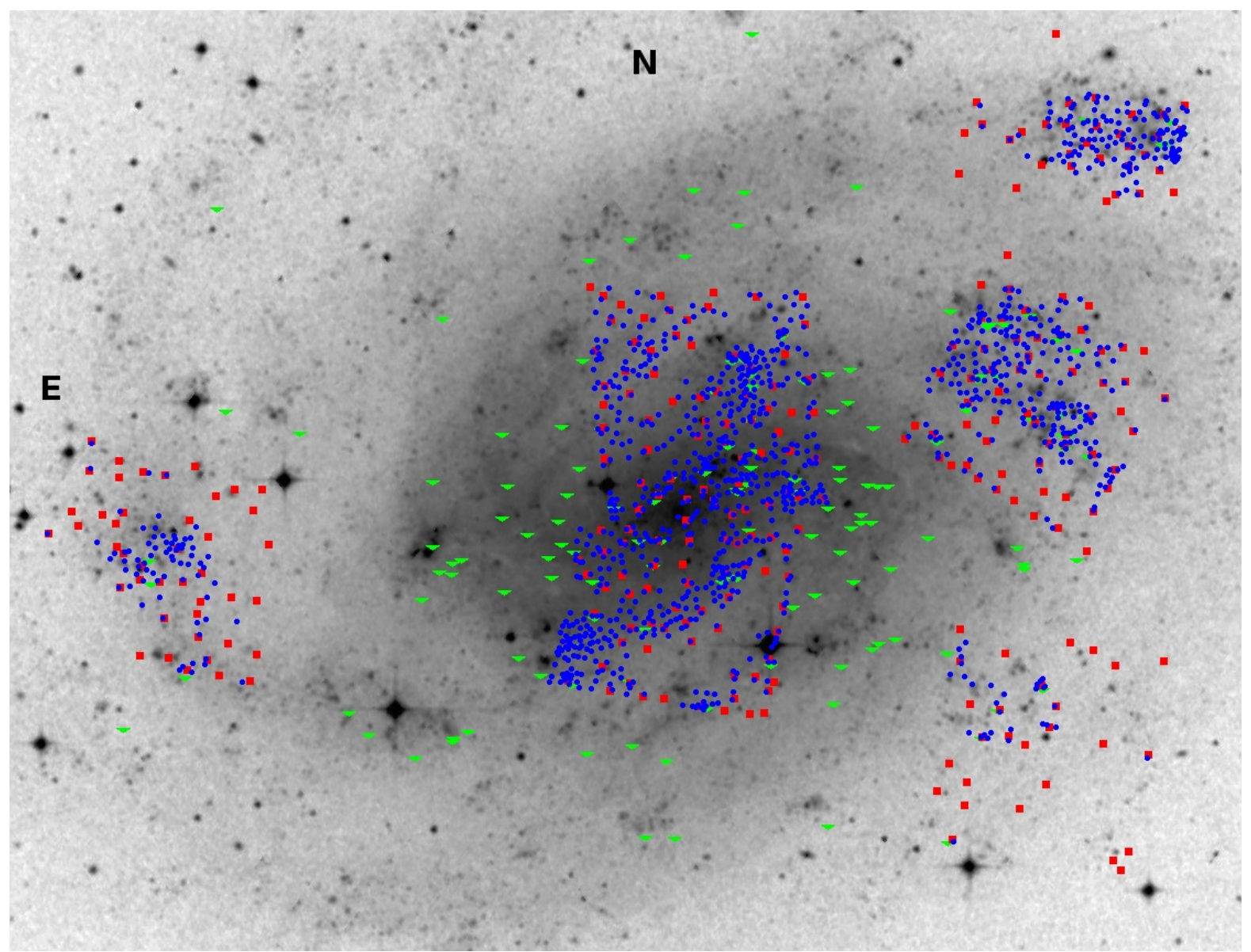

Fig. 5. Results obtained using SExtractor (red squares), PLC (blue circles), and the catalog obtained by Pietrzyński et al. (2001; green triangles) overlaid on a DSS image of NGC 300.

techniques and the results obtained by Pietrzyński et al. (2001) are shown in Fig. 5, in which we can see from the PLC results that the young stellar groups clearly delineate the spiral structure of the galaxy. The associations identified using the SExtractor code show a more sparse distribution because the group sizes are larger, since the pixels of the density maps are four times larger than the ACS/WFC. This causes several PLC detections to correspond to a single detection of SExtractor. This is a sign of the hierarchical distribution of the blue population in the galaxy, which we address in our discussion (see Sect. 6.2). For this reason and considering that we identified some spurious detections as border effects with SExtractor, we decided to adopt the PLC detections as the final catalog of young stars groups in the galaxy. In Table 2 we show the first ten rows of the catalog, the complete version is available at the CDS.

In Fig. 6 we show three PLC-detected associations at different galactocentric distances. In particular, association 4010 is located in the stellar complex AS002 named by Pietrzyński et al. (2001). As we show in Fig. 7, they detected four subgroups (a, b, c, and d). Using the ASC/HST observations and the PLC method, we detected at least 40 groups in the same region, which are indicated with blue squares in the figure.

\section{Analysis}

To perform a systematic, homogeneous, and efficient analysis of each identified group, we developed a dedicated numerical code in FORTRAN 95.

\subsection{Size and radial density profile}

The coordinates of the center and the sizes of the groups were estimated as the mean values and the dispersions of the positions of the blue bright group stars identified for each association.

The density profile was built by distinguishing the different color populations; these are the blue and red group. Then, the stellar density for each color was measured, considering concentric rings with a radial step of 0.4 arcsec. In Fig. 8 we show the radial density profile of association 4010 . A clear overdensity of blue stars is evident, while the density of red stars remains equal to zero. This is expected for a young stellar association.

\subsection{Field star decontamination}

Our code uses the coordinates of the previously localized groups and performs a statistical decontamination of the field stars based on the magnitudes and color indexes of the stars (see Gallart et al. 2003). The procedure consists of a star-by-star comparison of the quantities $F 555 W, F 435 W-F 555 W$ and $F 555 W-F 814 W$ between the stars of the group and the stars of a field region located near the association and covering the same sky area. Then, we subtracted the stars in the group that matched a star in the field region in these quantities. To obtain results as accurate as possible, the code carries out this procedure with five different field regions for each association. The first region is a ring around the association, and the others are selected as follows: $\left(\alpha_{0} \pm \Delta \alpha, \delta_{0}\right)$; $\left(\alpha_{0}, \delta_{0} \pm \Delta \delta\right)$ where $\Delta \alpha=\Delta \delta=20^{\prime \prime}$. We discarded the field regions with the largest and smallest number of stars to avoid 
Table 2. Young star groups in NGC 300.

\begin{tabular}{|c|c|c|c|c|c|c|c|c|c|c|c|c|c|c|c|c|}
\hline Name & Field & $\begin{array}{c}\text { raj2000 } \\
(\mathrm{deg})\end{array}$ & $\begin{array}{c}\text { dej2000 } \\
(\mathrm{deg})\end{array}$ & $r\left[{ }^{\prime \prime}\right]$ & $N$ & $N_{\text {bri }}$ & $N_{\text {dct }}$ & $N_{\text {dct-bri }}$ & $N_{\text {blue }}$ & $N_{\text {blue-dct }}$ & $N_{\text {red }}$ & $N_{\text {red-dct }}$ & $\mathrm{Mag}_{\min }$ & $\Gamma$ & $\operatorname{err}_{\Gamma}$ & $\begin{array}{c}d_{\mathrm{GC}} \\
{[\mathrm{Kpc}]}\end{array}$ \\
\hline 1001 & 1 & 13.890841 & -37.688836 & 0.57 & 12 & 7 & 11 & 7 & 10 & 10 & 1 & 0 & 21.52 & -0.20 & 0.31 & 4.94 \\
\hline 1002 & 1 & 13.933404 & -37.685159 & 0.38 & 10 & 10 & 10 & 10 & 7 & 7 & 0 & 0 & 23.46 & - & - & 6.27 \\
\hline 1003 & 1 & 13.890247 & -37.688886 & 0.78 & 23 & 6 & 20 & 6 & 12 & 10 & 5 & 4 & 22.60 & -0.00 & 0.80 & 4.93 \\
\hline 1004 & 1 & 13.904136 & -37.693602 & 1.46 & 53 & 9 & 40 & 8 & 32 & 25 & 13 & 9 & 21.09 & -0.87 & 0.29 & 5.28 \\
\hline 1005 & 1 & 13.894911 & -37.690068 & 1.50 & 53 & 9 & 36 & 6 & 30 & 21 & 16 & 11 & 23.30 & - & - & 5.05 \\
\hline 1006 & 1 & 13.892505 & -37.685818 & 1.22 & 34 & 8 & 26 & 6 & 21 & 16 & 3 & 3 & 21.53 & -0.50 & 0.42 & 5.04 \\
\hline 1007 & 1 & 13.892056 & -37.689406 & 1.00 & 33 & 6 & 26 & 5 & 18 & 14 & 8 & 6 & 22.93 & - & - & 4.97 \\
\hline 1008 & 1 & 13.886917 & -37.690713 & 2.44 & 153 & 14 & 89 & 11 & 77 & 46 & 46 & 25 & 21.55 & -1.19 & 0.33 & 4.80 \\
\hline 1009 & 1 & 13.889945 & -37.697110 & 1.71 & 69 & 8 & 48 & 8 & 37 & 26 & 10 & 6 & 21.25 & -0.29 & 0.23 & 4.82 \\
\hline 1010 & 1 & 13.884190 & -37.692673 & 1.50 & 62 & 8 & 41 & 6 & 30 & 20 & 20 & 12 & 23.80 & - & - & 4.70 \\
\hline
\end{tabular}

Notes. The suffix $b r i$ indicates bright stars with $F 555 W<25$. The suffix $d c t$ indicate stars belonging to the decontaminated region. The suffixes blue and red refer to blue and red stars respectively. $\mathrm{Mag}_{\min }$ is the magnitude of the brightest star in the group. $\Gamma$ is the PDMF slope. $d_{\mathrm{GC}}$ is the galactocentric distance. Here we present the first ten rows, the complete table is available at the CDS.

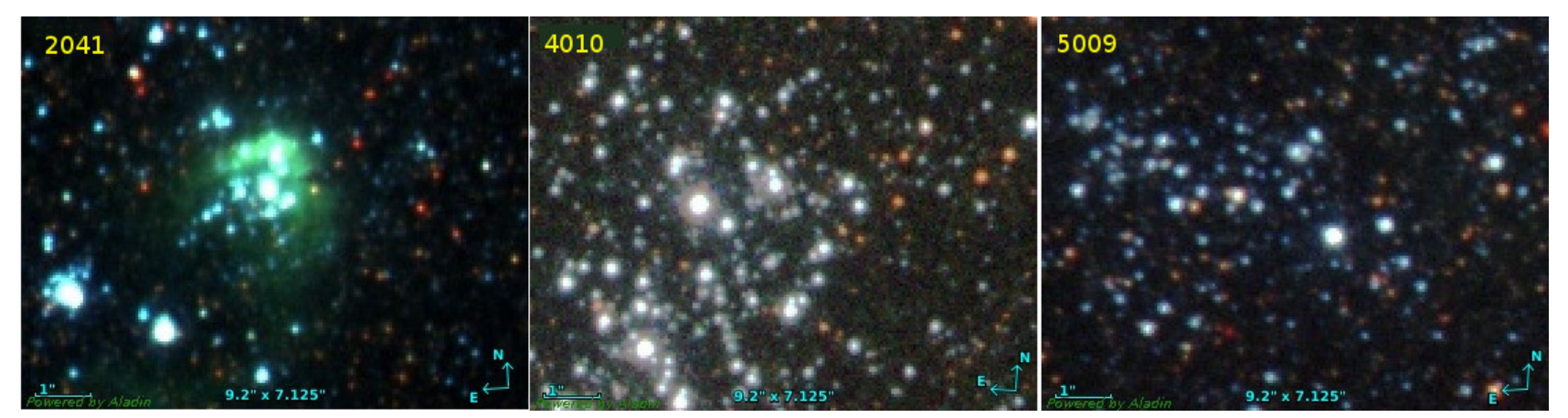

Fig. 6. Color images of the associations 2041, 4010, and 5009 located at different galactocentric distances. These groups were detected with the PLC method.

possible neighboring associations or to fall outside the field of view. The final parameters of an association are an average of the parameters obtained with the three remaining field regions.

From these results we built the photometric diagrams and mass histograms and calculated the fundamental parameters of each group in this way.

\subsection{Color-magnitude diagrams}

We used the blue and red group to build the color-magnitude diagrams (CMDs) shown in Fig. 9. The evolutionary models of Marigo et al. (2008) with the Girardi et al. (2010) corrections, corresponding to different ages and metallicities, were superposed for comparison. To displace the models and compare them with our data, we adopted a distance modulus of $\left(V o-M_{V}\right)=26.43$ (Bresolin et al. 2005), a normal reddening law $\left(R=A_{V} / E(B-V)=3.1\right)$, and a value for $E(B-V)=0.075$, where the Galactic foreground reddening toward NGC 300 of $0.025 \mathrm{mag}$ and an additional reddening of $E(B-V)=0.05 \mathrm{mag}$ intrinsic to NGC 300 were considered (Gieren et al. 2004).

The obtained CMDs for most of the identified young star groups revealed a sharp distribution of blue stars. This fact was understood as a consequence of a very low reddening inside the galaxy (Gieren et al. 2004). This situation eliminates possible degeneracy between temperature and extinction for hot stars. Then, as in the following section, we were able to estimate stellar masses from their magnitudes and compare them with fitted theoretical evolutionary models.

\subsection{Masses and the present-day mass function}

With the purpose of deriving stellar masses, we performed a lineal interpolation in the $F 555 \mathrm{~W}$ band in the adopted evolutionary models of 1 Myr (see Sect. 4). To avoid foreground stars in each association, we estimated the stellar masses of the blue-group stars resulting from the statistical decontamination. Given that NGC 300 presents an abundance gradient with $Z$ between 0.004-0.018 (Bresolin et al. 2009; Gazak et al. 2015), we tried to estimate the masses with a range of metallicities between $Z=0.007$ and $Z=0.019$. Finally, we adopted solar metallicity, since we did not obtain significant differences. The corresponding obtained mass histograms are presented in Fig. 10. Then, using only those bins corresponding to the highmass range $\left(M>7.4 M_{\odot}\right.$, which corresponds to the blue bright group), we performed linear fits to the data. These histograms are representative of the corresponding present-day mass functions (PDMFs). Additionally, in the adopted mass range, the PDMF can be modeled as a power law and expressed in the form $\log (N / \Delta(\log m))=\Gamma \Delta(\log m)$, where $N$ is the number of stars per logarithmic mass bin $\log (m)$. The computed corresponding slope values $(\Gamma)$ are also indicated in Fig. 10, and the obtained PDMF slopes for each detected association are presented in Table 2.

To estimate the effect of galaxy distance uncertainty and/or possible variable extinction in our procedure, we repeated it for different distance values (26.43 \pm 0.09 , Bresolin et al. 2005) and using $A_{V}$ values ranging from 0 to 0.53 (Roussel et al. 2005). We obtained that the changes in stellar mass are $\Delta M / M \sim 25 \%$ and changes in the corresponding PDMF slopes are lower than the 


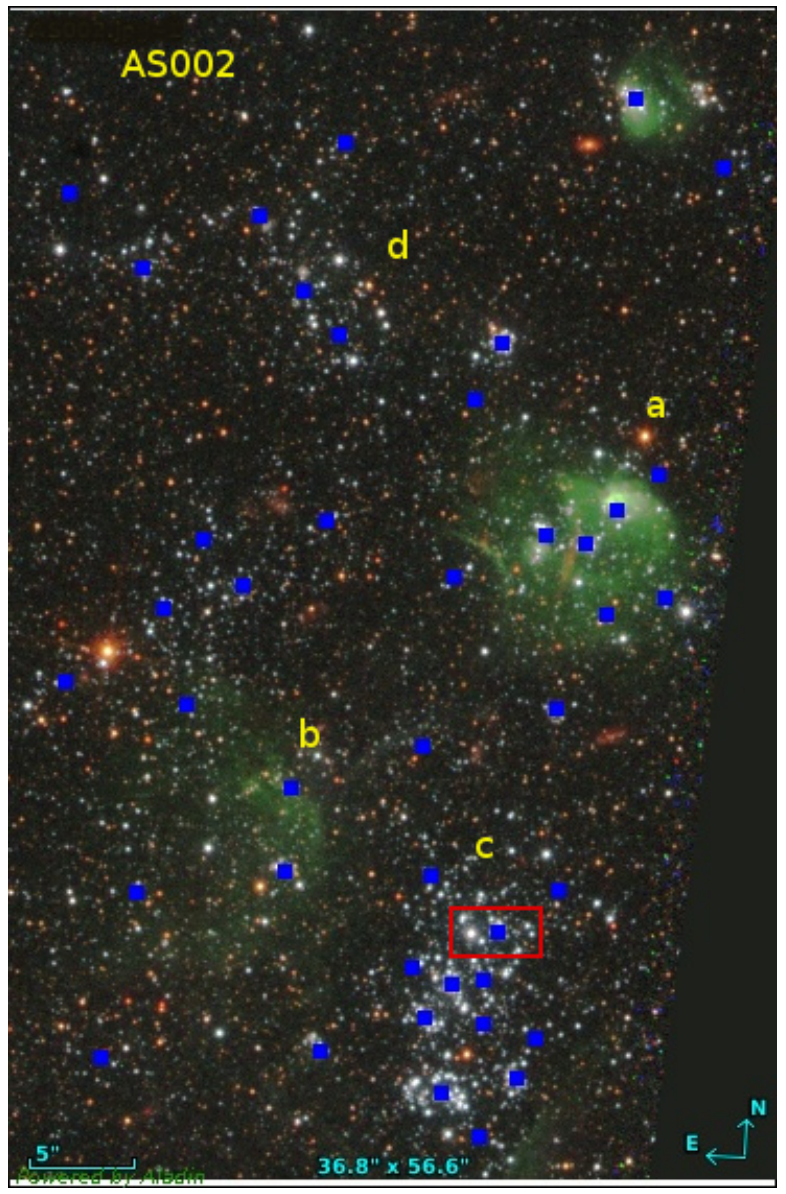

Fig. 7. Stellar complex AS002 named by Pietrzyński et al. (2001). The letters a to d designate the four subgroups detected in their work. Our PLC detections are indicated with blue squares. The red rectangle encloses the association 4010, which is shown in Fig. 6.

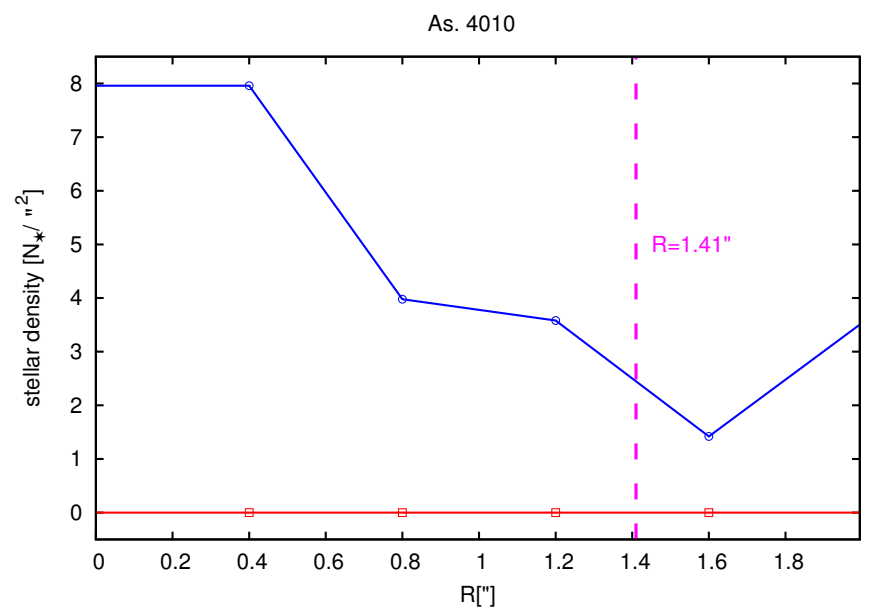

Fig. 8. Radial density profile of association 4010. With blue and red we indicate the density of blue and red group stars; the pink dashed line shows the adopted association radius at $1.41^{\prime \prime}$.

indicated fitted errors. Therefore, our analysis and conclusions are independent of the above indicated effects.
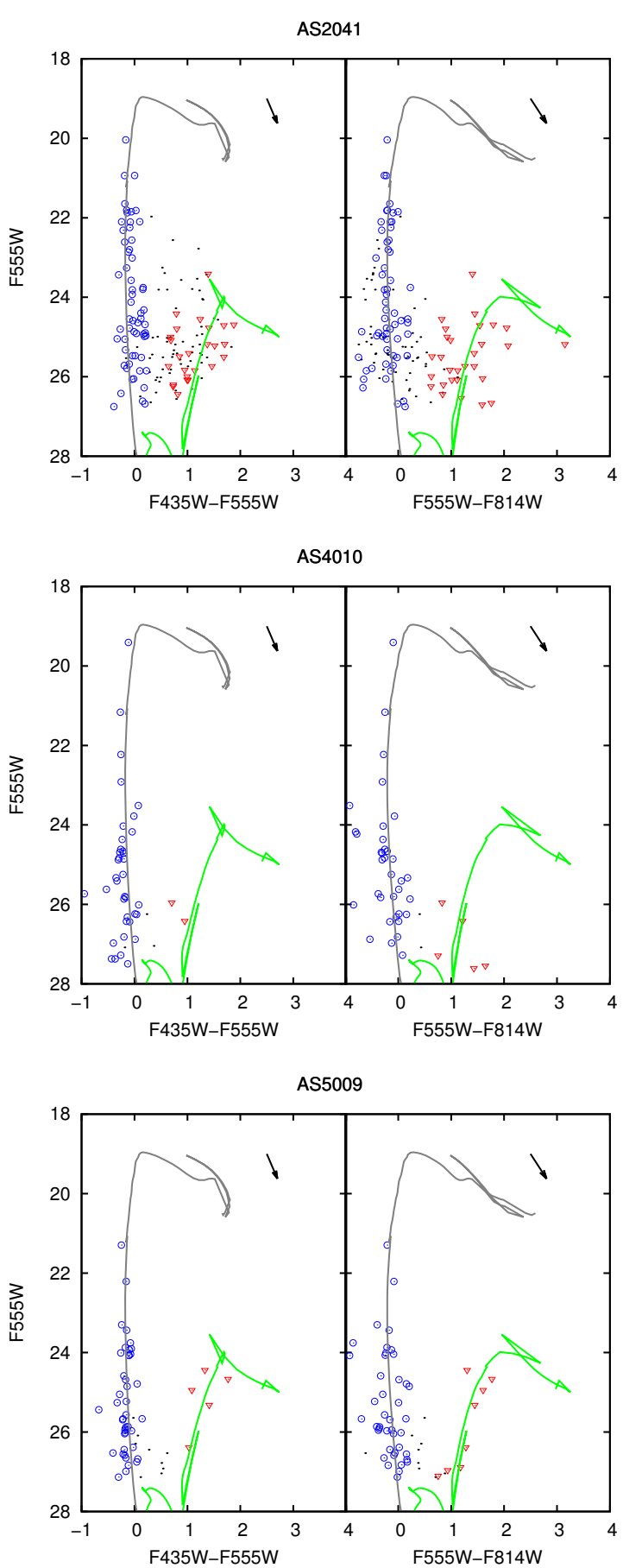

Fig. 9. Decontaminated color-magnitude diagrams $F 555 W$ vs. $F 435 W-F 555 W$ and $F 555 W$ vs. $F 555 W-F 814 W$ of the stellar associations 2041, 4010, and 5009 that are located in different places of the galaxy. The blue circles represent the blue group of stars, the red inverted triangle indicate the red group, and the black dots are the stars that are neither blue nor red. The gray line indicates the isochrone corresponding to $10^{7} \mathrm{yr}$ and solar metallicity $(Z=0.019)$. The green line corresponds to $10^{9} \mathrm{yr}$ and $Z=0.008$ (Marigo et al. 2008; Girardi et al. 2010). The arrow indicates the reddening vector.

\section{Discussion: Global properties of the young star groups}

\subsection{Size distribution}

From the size distribution of the 1147 young star group detected using the PLC method shown in Fig. 11, we derived 



Fig. 10. Present-day mass function of the stellar associations 2041, 4010 , and 5009 that are located in different places of the galaxy. The line corresponds to a linear fit over the gray bins $\left(M>7.4 M_{\odot}\right)$.

that the average radius and the highest value in the distribution are both $\sim 2.5$ arcsec. At the considered distance this is approximately equivalent to $25 \mathrm{pc}$. This value is lower than the one found by Pietrzyński et al. (2001) of 57 pc. This difference is expected since the HST/ACS images used in this work have higher resolution than the ground-based images. For this reason, they applied the PLC method with a parameter of distance $d_{\mathrm{s}}$ much greater than ours $(7,7.5$ and 8 arcsec). Bresolin et al. (1998) performed a study of OB association for seven spiral galaxies observed with the HST (NGC 925, NGC 2090, NGC 2541, NGC 3351, NGC 3621, NGC 4548, and M101), finding that the size distribution peaked between radius of $20-40 \mathrm{pc}$, which is consistent with our value. Pietrzyński et al. (2005) compared these data with several catalogs for OB associations in nearby galaxies in the Local Group (IC 1613, LMC, M 33, NGC 6822, LMC, SMC, and M 31) and some more distant galaxies (UGC 12732, NGC 1058, NGC 7217, NGC 4394,

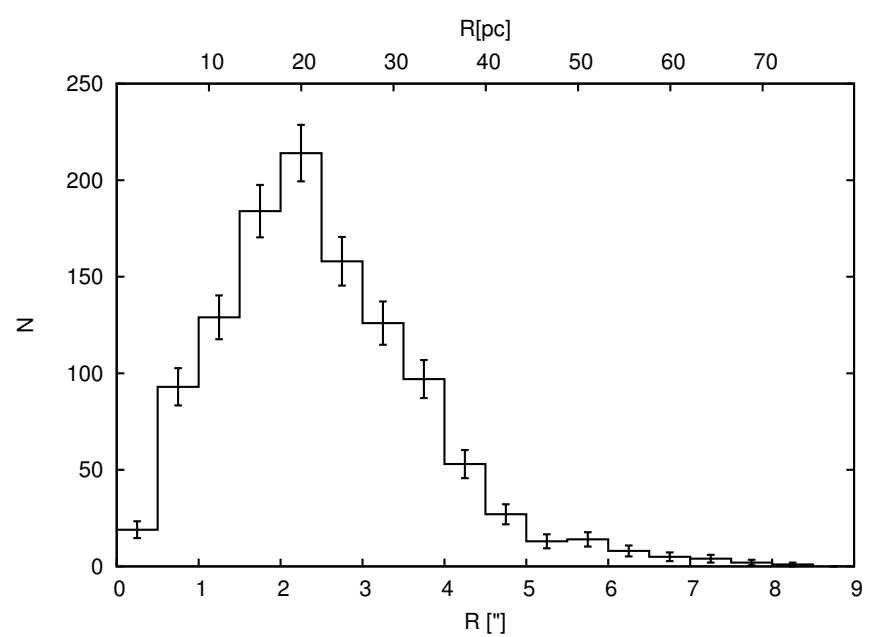

Fig. 11. Size distribution of young star groups in NGC 300.

NGC 3507, and NGC 337A). They found a peak in the size distribution between $25-55 \mathrm{pc}$ and an average radius between 20-90 pc for these galaxies. These values agree well with our results.

\subsection{Hierarchical structure in NGC 300}

It is known that young stars cluster tend to be part of larger groups, such as stellar complexes, which are grouped themselves in larger structures. While we searched for the best-fit distance parameter for the PLC, it became evident that the blue stellar population in NGC 300 presents a hierarchical structure, which starts with the spiral arms and concluds with individual compact clusters, or even with multiple stars. To show this, we traced contours at different density levels on our density stellar maps, created as described in Sect. 4.1. In Fig. 12 we show the different density contours applied to the density maps of the central fields (fields 2 and 3) for the blue bright (left panel) and the red bright group (right panel). The isocontours were created using the tool cont of ALADIN with four different density values (40, 80,110 , and 145 stars per bin of $8 \operatorname{arcsec}^{2}$ ); this means different density levels in the density stellar maps. It is clear from Fig. 12 that the blue population follows the spiral arm structure of the galaxy, while the red population is concentrated in the galaxy bulge. The left panel shows several young structures at different density levels. The denser smaller concentrations tend to belong to larger and looser ones, pointing a hierarchical structure in the distribution of blue stars in this galaxy. To easily visualize the ties among structures of different density levels, we built a tree diagram; these types of diagrams are called dendrograms (Gouliermis et al. 2010) and indicate the connection between parent and child structures corresponding to lower and higher density levels, respectively. In Fig. 13 we show the dendrograms of the blue stellar structures identified in the four density levels mentioned above. We added a last level for the most compact structures detected using PLC. The pixels size of the density maps is 2 arcsec, therefore it is not possible to detect the smallest groups that are effectively detected with PLC.

\subsection{PDMF behavior}

Several works have claimed a possible relation between the PDMF shape and the galactocentric distance in galaxies (e.g., Martín-Navarro et al. 2015), since its trend would depend on 


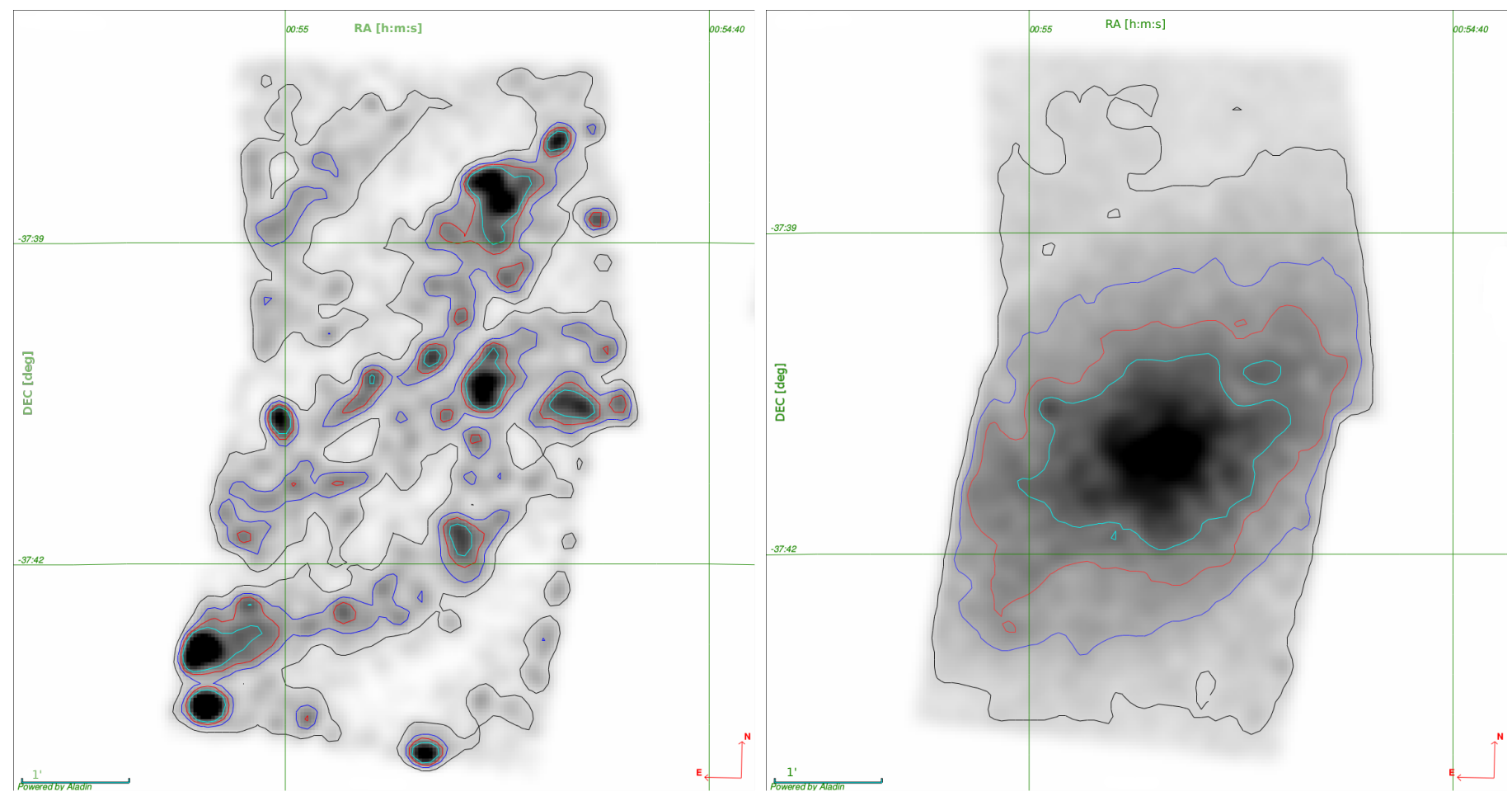

Fig. 12. Density maps of central fields for the blue bright group (left panel) and the red bright group (right panel). The overlapping contours



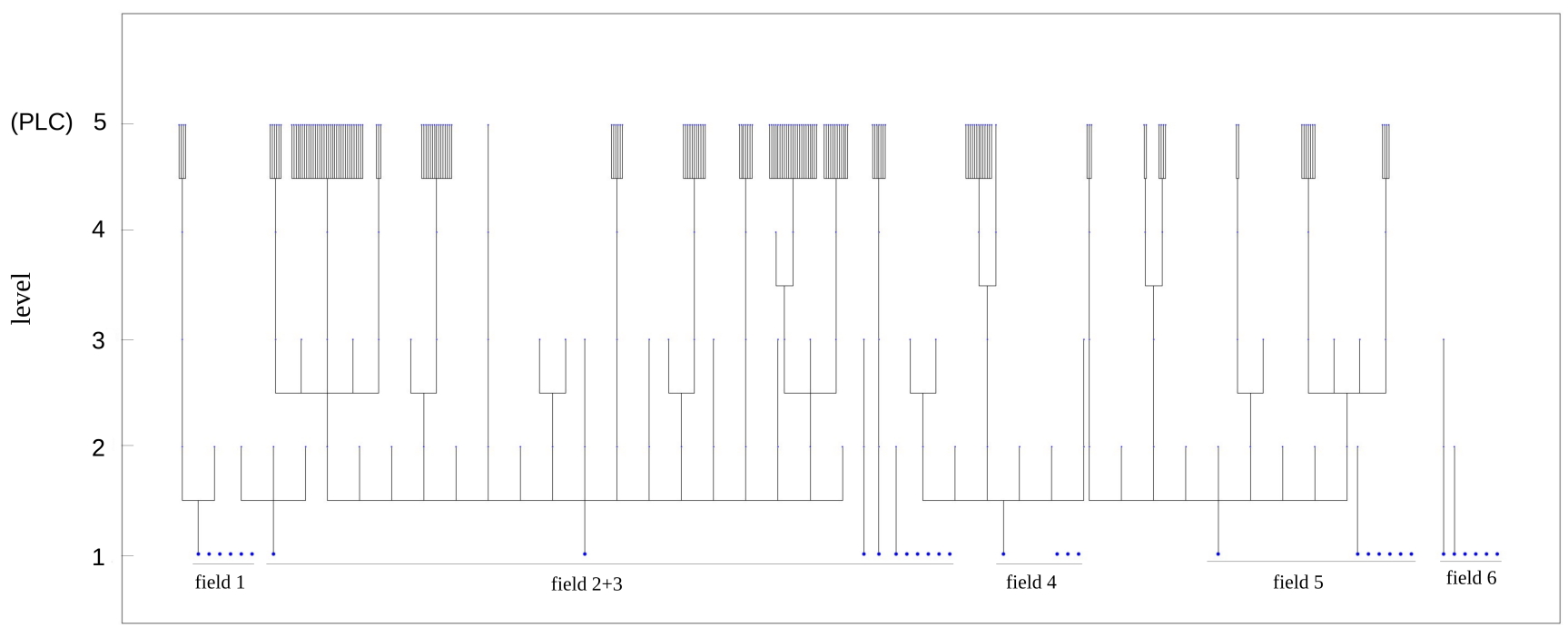

Fig. 13. Dendrogram of the young stellar structures detected at different density level. Levels 1-4 correspond to a pixel value in the density maps of $40,80,110$, and 145, respectively. Level 5 corresponds to the most compact cluster detected using PLC. The blue circles at the bottom indicate the structures detected at the lower density level. Some of them are no longer detected at higher densities.

the local properties within a galaxy. Searching for a possible relation of this kind in NGC 300, we derived the galactocentric distance of each stellar group considering the geometrical parameters of the galaxy given by Puche et al. (1990). These are a position angle $\gamma=114.3^{\circ}$ and an inclination $i=50^{\circ}$. To obtain a more reliable result, we only considered the most relevant stellar groups found in NGC 300, that is, those with the lowest PDMF slope errors $\left(e_{\Gamma}<0.5\right)$, with massive stars $\left(M>25 M_{\odot}\right)$, and a large number of blue stars $(N>100)$. Our sample of stellar groups was then 25, and they are plotted in Fig. 14. It seems there is a slight trend for flatter slopes in the outside limit of the galaxy than in the central region (left panel). We found a mean slope of $-1.3 \pm 0.1$ for $d_{\mathrm{GC}}<2 \mathrm{kpc}$ and $-0.9 \pm 0.2$ for $d_{\mathrm{GC}}>4 \mathrm{kpc}$. However, within the errors, we are unable to confirm a clear dependence of the $\Gamma$ slope with the galactocentric distance for NGC 300. We can only indicate their distribution (right panel) and their average and standard deviation (s.d.) values as -1.16 and 0.39 , respectively. 


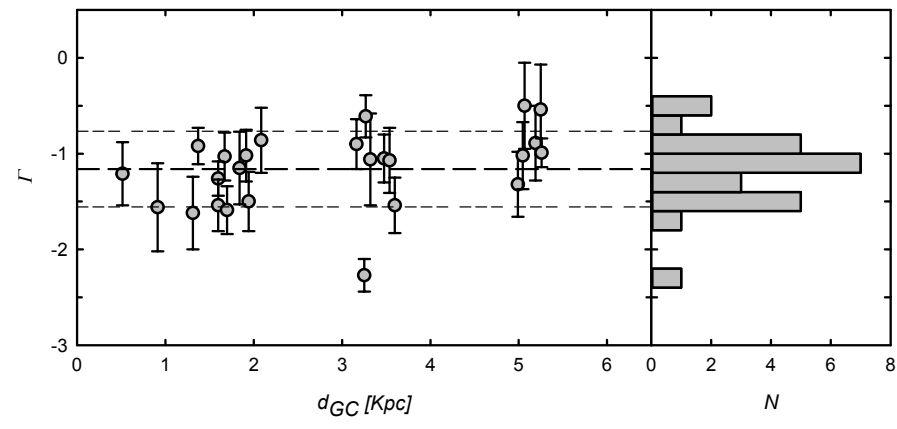

Fig. 14. PDMF behavior for the most important groups found in NGC 300 (see text for details). The long dashed line indicates the mean slope value, and the short dashed lines show the envelopes at one standard deviation.

\section{Conclusions}

Using complementary methods for the detection of young stellar groups in NGC 300, we found that the blue population is located in the spiral arms of the galaxy, presenting a hierarchical behavior where denser and smaller structures are enclosed in larger and less dense ones. We were able to delineate the hierarchical structure on several density levels.

Through the PLC technique, 1147 young stellar groups have been detected in six fields of the galaxy NGC 300. The resulting catalog contains the coordinates of the groups, sizes, number of stars, PDMF slope with its error, and galactocentric distance. In Table 2 we present the first ten rows. The completed catalog is available at the CDS. The identification name of each grouping consists of four numbers: the first is the field number, 1, 2, 4, 5 , or 6 , because fields 2 and 3 were joined into a single field denoted by number 2 (see Sect. 3.3), and the remaining three numbers indicate the number of group in the field.

The mode and the mean of the groups size distribution are both $25 \mathrm{pc}$. These values agree well with those found in the literature for the Local Group and some more distant galaxies. We did not find a clear dependence of the $\Gamma$ slope on galactocentric distance for the PDMF of the most important groups, but a slight trend seems to exist that deserves a more detailed analysis. We can only indicate that the mean value is $-1.16 \pm 0.39$ (s.d.). This slope is slightly flatter than the -1.35 value given by Salpeter (1955) but is compatible within the errors.

Acknowledgements. We thank the referee for helpful comments and constructive suggestions that helped to improve this paper. M.J.R. and G.B. acknowledge support from CONICET (PIP 112-201101-00301). M.J.R. is a fellow of CONICET. Based on observations made with the NASA/ESA Hubble Space Telescope, and obtained from the Hubble Legacy Archive, which is a collaboration between the Space Telescope Science Institute (STScI/NASA), the Space Telescope European Coordinating Facility (ST-ECF/ESA) and the Canadian Astronomy Data Centre (CADC/NRC/CSA). Some of the data presented in this paper were obtained from the Mikulski Archive for Space Telescopes (MAST). STScI is operated by the Association of Universities for Research in Astronomy, Inc., under NASA contract NAS5-26555. Support for MAST for non-HST data is provided by the NASA Office of Space Science via grant NNX09AF08G and by other grants and contracts. This research has made use of "Aladin sky atlas" developed at CDS, Strasbourg Observatory, France. Finally we would like to thank to Dr. Guido Moyano Layola, the English cabinet of FCAG-UNLP, and the language editor Astrid Peter for English corrections.

\section{References}

Battinelli, P. 1991, A\&A, 244, 69

Bresolin, F., Kennicutt, Jr., R. C., Ferrarese, L., et al. 1998, AJ, 116, 119

Bresolin, F., Pietrzyński, G., Gieren, W., \& Kudritzki, R.-P. 2005, ApJ, 634, 1020

Bresolin, F., Gieren, W., Kudritzki, R.-P., et al. 2009, ApJ, 700, 309

Dalcanton, J., Williams, B., \& ANGST Collaboration 2008, The ACS Nearby

Galaxy Survey Treasury: 9 Months of ANGST, eds. H. Jerjen, \& B. S. Koribalski, 115

Dolphin, A. E. 2000, PASP, 112, 1383

Fruchter, A. S., \& Hook, R. N. 2002, PASP, 114, 144

Gallart, C., Zoccali, M., Bertelli, G., et al. 2003, AJ, 125, 742

Gazak, J. Z., Kudritzki, R., Evans, C., et al. 2015, ApJ, 805, 182

Gieren, W., Pietrzyński, G., Walker, A., et al. 2004, AJ, 128, 1167

Girardi, L., Williams, B. F., Gilbert, K. M., et al. 2010, ApJ, 724, 1030

Gouliermis, D. A., Schmeja, S., Klessen, R. S., de Blok, W. J. G., \& Walter, F. 2010, ApJ, 725, 1717

Marigo, P., Girardi, L., Bressan, A., et al. 2008, A\&A, 482, 883

Martín-Navarro, I., Barbera, F. L., Vazdekis, A., Falcón-Barroso, J., \& Ferreras, I. 2015, MNRAS, 447, 1033

McConnachie, A. W. 2012, AJ, 144, 4

Pietrzyński, G., Gieren, W., Fouqué, P., \& Pont, F. 2001, A\&A, 371, 497

Pietrzyński, G., Ulaczyk, K., Gieren, W., Bresolin, F., \& Kudritzki, R. P. 2005, A\&A, 440, 783

Puche, D., Carignan, C., \& Bosma, A. 1990, AJ, 100, 1468

Roussel, H., Gil de Paz, A., Seibert, M., et al. 2005, ApJ, 632, 227

Salpeter, E. E. 1955, ApJ, 121, 161 